\title{
Dendritic Computation of Direction Selectivity and Gain Control in Visual Interneurons
}

\author{
Sandra Single, Juergen Haag, and Alexander Borst \\ Friedrich-Miescher-Laboratory, Max-Planck-Society, D-72076 Tuebingen, Germany
}

The extraction of motion information from time varying retinal images is a fundamental task of visual systems. Accordingly, neurons that selectively respond to visual motion are found in almost all species investigated so far. Despite its general importance, the cellular mechanisms underlying direction selectivity are not yet understood in most systems. Blocking inhibitory input to fly visual interneurons by picrotoxinin (PTX), we demonstrate that their direction selectivity arises largely from interactions between postsynaptic signals elicited by excitatory and inhibitory input elements, which are themselves only weakly tuned to opposite directions of motion. Their joint acti- vation by preferred as well as null direction motion leads to a mixed reversal potential at which the postsynaptic response settles for large field stimuli. Assuming the activation ratio of these opponent inputs to be a function of pattern velocity can explain how the postsynaptic membrane potential saturates with increasing pattern size at different levels for different pattern velocities ("gain control"). Accordingly, we find that after blocking the inhibitory input by PTX, gain control is abolished.

Key words: direction selectivity; motion detection; membrane parameters; compartmental model; synaptic conductance; neural computation; gain control; fly
The fly has for long been a model system to study the processing and extraction of motion information from the time varying retinal images. In the third visual neuropile of the fly optic lobes a group of individually identifiable, motion-sensitive interneurons has been found. They are called lobula plate tangential cells (LPTCs) and are involved in visual course control (Hausen, 1984). In the blowfly Calliphora erythrocephala this group comprises about 60 different cells. Via large dendritic arbors, these neurons spatially pool the signals of thousands of retinotopically arranged columnar elements (Borst and Egelhaaf, 1992). Most LPTCs studied so far display a directionally selective response: When the pattern is moving in the preferred direction (PD) of the cell, the cells become excited; when the pattern is moving in the anti-preferred or null direction (ND) of the cell, they become inhibited. Many LPTCs are nonspiking neurons. Rather than producing regular action potentials, they respond to visual motion by a graded shift of their membrane potential. Their directional selective responses are driven by at least two kinds of input elements, one being excitatory and the other inhibitory (Borst and Egelhaaf, 1990; Borst et al., 1995) (Fig. 1a). As was revealed by in vitro studies (Brotz et al., 1995), the underlying dendritic receptors exhibit a pharmacological profile typical for insect nicotinic ACh receptors and picrotoxinin (PTX)-sensitive GABA receptors, respectively (Brotz and Borst, 1996).

The computational structure of the fly motion detection system can be well described by a correlation type of elementary motion detector (EMD) (Borst and Egelhaaf, 1989; Egelhaaf and Borst, 1989). This model for motion detection assumes a delay-and-

Received April 15, 1997; revised May 22, 1997; accepted May 23, 1997.

We are grateful to T. Martin for excellent technical assistance, T. Brotz, V. Gauck, J. Gold, and F. Theunissen for helpful discussions and critically reading earlier versions of this manuscript, and K. G. Goetz (Max-Planck-Institute of Biological Cybernetics) for the generous loan of part of the equipment.

Correspondence should be addressed to Alexander Borst, Friedrich-MiescherLaboratory, Max-Planck-Society, Spemannstrasse 37-39, D-72076 Tuebingen, Germany.

Copyright (C) 1997 Society for Neuroscience $\quad 0270-6474 / 97 / 176023-08 \$ 05.00 / 0$ compare mechanism for each retinal location; the local luminance value as measured at one retinal location is delayed or low-passfiltered and subsequently multiplied with the instantaneous luminance value as derived from a neighboring location (Fig. 1b). When two such elements are taken, one being the mirror image of the other, a fully directional local signal is obtained by subtracting their output signals from each other. For visual course control, the output signals of many of such local units are assumed to be spatially integrated in an appropriate way. Although this computational model can explain many specific features of the visual response properties of the fly LPTCs, the actual implementation at the cellular level is not known so far. In particular, the degree of direction selectivity, being carried by the LPTC input elements, is still an open question (Douglass and Strausfeld, 1995; Douglass and Strausfeld, 1996). If on one hand, the input elements show a strong direction selectivity, the dendrite of the LPTCs would exclusively serve to integrate the local motion signals spatially. On the other hand, the input elements could reveal a weak directional tuning, and the dendrite of the LPTCs would then, in addition to integrate the inputs spatially, also enhance direction selectivity through the opponent action of these input elements. In terms of the computational model, the question is whether the subtraction of the mirror symmetrical units takes place presynaptic to the LPTCs or directly on the dendrites of LPTCs. Although this makes no difference in a model with linear spatial summation, both design principles result in different response properties when the physiological spatial integration properties of real dendrites are taken into account.

\section{MATERIALS AND METHODS}

\section{Animal preparation}

Female blowflies (Calliphora erythrocephala) were briefly anesthesized with $\mathrm{CO}_{2}$ and mounted ventral side up with wax on a small preparation platform. The head capsule was opened from behind; the trachea and air sacs, which normally cover the lobula plate, were removed. To eliminate movements of the brain caused by peristaltic contractions of the esophagus, the proboscis of the animal was cut away, and the gut was pulled 
out. This allowed stable intracellular recordings of up to $45 \mathrm{~min}$. The fly was then mounted in an upright position on a heavy recording table with the stimulus monitors in front of the animal. The fly brain was viewed from behind through a Zeiss dissection scope. For details of the dissection procedure, see Hausen (1982).

\section{Intracellular recording}

Electrophysiology. Recording electrodes were made of glass capillaries (GC100TF-10; Clark Electromedical Instruments, Pangbourne, UK) pulled on a Brown-Flaming P87 puller; when filled with $2 \mathrm{M}$ KAc and 0.5 $\mathrm{M} \mathrm{KCl}$ they had a resistance of about $20 \mathrm{M} \Omega$. Signals were amplified (SEC-10L; npi Electronics; operated in DCC mode at a switching frequency of $20 \mathrm{kHz}$ ) and fed to a 486 personal computer (PC) via an analog-to-digital (A/D) converter (DT 2801-A; Data Translation) at 2 $\mathrm{kHz}$.

Stimulation. Stimuli were generated on a Tektronix 608 monitor by an image synthesizer (Picasso, Innisfree, Cambridge, MA) and consisted of a one-dimensional square wave grating of $28^{\circ}$ spatial wavelength, $84 \%$ contrast, and $11.9 \mathrm{~cd} / \mathrm{m}^{2}$ mean luminance displayed at a frame rate of 200 $\mathrm{Hz}$. The angular width of the stimulus field was $62^{\circ}$ in the horizontal and $74^{\circ}$ in the vertical direction as seen by the fly. When activated, the grating was moving at $56^{\circ} / \mathrm{sec}$.

Stimulus protocol and data processing. Each cell was continuously subject to the stimulus protocol shown in Figure $2 a$. For $2 \mathrm{sec}$, the pattern was at rest; then, the cell was stimulated for $2 \mathrm{sec}$ by motion in its preferred direction and, for another $2 \mathrm{sec}$, by motion in its null direction. This sequence was repeated over and over again. For each stimulus condition (no motion, PD motion, and ND motion), five pulses of $2 \mathrm{nA}$ of hyperpolarizing current were injected into the cell to determine the input resistance $\left(R_{\mathrm{IN}}\right)$. The motion-induced change of input resistance, $\left[R_{\mathrm{IN}}(\right.$ motion $)-R_{\mathrm{IN}}($ rest $\left.)\right] / R_{\mathrm{IN}}($ rest $) \times 100$, was calculated within each sweep from averaging the responses to the five current pulses. The values derived from several sweeps were subsequently averaged within each experiment. After 5-15 sweeps, $1 \mu \mathrm{l}$ of a $10^{-4} \mathrm{M}$ solution of PTX diluted in fly saline was injected into the hemolymph close to the lobula plate by means of a syringe. To allow for rapid diffusion, the neurolemma covering the lobula plate was punctuated before by means of a tungsten electrode. The responses were recorded for about 15-30 min. Because the time course of the PTX effect varied from recording to recording, averages were taken of those response sweeps for which the ND responses had reversed their sign and amounted to at least $50 \%$ of the PD response.

\section{Extracellular recording}

Electrophysiology. Extracellular recordings were performed from the axonal arborizations of the $\mathrm{H} 1$-cell in the hemisphere contralateral to the stimulus side using a sharpened tungsten electrode. We decided to record from this spiking neuron, because experiments lasting several hours can easily be accomplished in extracellular recordings, and the general visual response characteristics of the H1-cell do not differ from those of VSand $\mathrm{CH}$-cells (Hausen, 1984). Long recording times were necessary to ensure ample control measurements such as, for example, the spatial sensitivity distribution for PD and ND motion at both velocities. Signals were bandpass-filtered, transformed into discrete pulses by means of a window discriminator, and counted in $1 \mathrm{sec}$ bins before feeding them to a 486 PC via an A/D converter (DT 2801-A; Data Translation) at $1 \mathrm{~Hz}$.

Stimulation. Stimuli consisted of a one-dimensional sine wave grating of $24^{\circ}$ spatial wavelength, $29 \%$ (Fig. $3 c, d$ ) and $8 \%$ (Fig. 3e,f) contrast, and $21 \mathrm{~cd} / \mathrm{m}^{2}$ mean luminance displayed at a frame rate of $200 \mathrm{~Hz}$. The angular width of the stimulus field was $58^{\circ}$ in the horizontal direction and $43^{\circ}$ in the vertical direction as seen by the fly. Pattern size was varied in four or five steps of 10.7 or $8.6^{\circ}$, respectively, along the vertical axis of the stimulus monitor and presented at two different velocities for PD motion (72 or $360^{\circ} / \mathrm{sec}$ ).

Stimulus protocol and data processing (different from intracellular recordings). PTX was diluted in fly saline to $3 \times 10^{-4} \mathrm{M}$ concentration and applied in two different ways: either it was added to the hemolymph after punctuating the lobula plate (same as for intracellular recording); or it was pressure-injected directly into the lobula plate. Both application methods yielded similar results. Responses were recorded for $\sim 2-3 \mathrm{hr}$.

\section{Simulations}

Denoting the spatial separation between the input lines of the motion detectors as $\Delta \varphi$, the pattern had a one-dimensional sinusoidal luminance modulation of wavelength $\lambda=16 \Delta \varphi$ with a contrast of $90 \%$. Each of the 32 EMDs was built from two input sensors measuring the local luminance values, first order temporal low-pass filters with a time constant $\tau=60 \mathrm{msec}$, and multipliers $M$. The output signals of the motion detectors were used as excitatory and inhibitory conductances (about 1-2 $\mathrm{mS} / \mathrm{cm}^{2}$, the exact value depending on the stimulus condition) for a variable number of dendritic membrane areas of the cell, using the compartmental model software Nemosys (Eeckman et al., 1994). The membrane parameters were assumed to be spatially uniform with $R_{\mathrm{m}}=$ $2 \mathrm{k} \Omega \mathrm{cm}^{2}, R_{\mathrm{i}}=40 \Omega \mathrm{cm}$, and $C_{\mathrm{m}}=0.8 \mu \mathrm{F} / \mathrm{cm}^{2}$. The synaptic reversal potentials, relative to the $E_{\text {leak }}$, were $E_{\text {exc }}=+40 \mathrm{mV}$, and $E_{\mathrm{inh}}=-30$ $\mathrm{mV}$ for weak directional EMDs (model 1); and $E_{\mathrm{exc}}=+24 \mathrm{mV}$ and $E_{\mathrm{inh}}=-13 \mathrm{mV}$ for strong directional EMDs (model 2). For testing the spatial integration properties, four dendritic membrane areas were distributed between the top and bottom of the main dendrite. Each area comprised only small dendritic branchlets $(<4 \mu \mathrm{m})$ and had a membrane area between 31 and $70 \times 10^{-6} \mathrm{~cm}^{2}$. The areas were set to roughly yield the same axonal membrane potential when stimulated with identical conductance changes. For further details of the passive membrane properties of the tangential cells, see Borst and Haag (1996).

\section{RESULTS}

We investigated the degree of direction selectivity of the input elements of LPTCs by a combined experimental and modeling approach. Our experimental diagnostics consisted in intracellular recordings of the motion response of LPTCs as well as in measurements of their motion-induced change of input resistance $\left(R_{\mathrm{IN}}\right)$, a measure of synaptic conductance changes. We also disturbed the system by blocking inhibitory input synapses with PTX. As will be shown by our simulation studies, both weak and strong directional EMDs can lead to identical reactions of the LPTCs. However, when inhibitory synapses are blocked, the two alternatives then lead to different predictions and, thus, allow to decide between them experimentally.

The simulation study comprised two arrays of EMDs tuned to opposite directions of motion, which make excitatory and inhibitory synapses on the dendrite of a realistic compartmental model of a fly LPTC, respectively (Fig. 1a). The model cell had only passive membrane properties, the precise values of which were determined in an independent set of experiments (Borst and Haag, 1996). We modeled two different types of EMDs: one type with a weak (Fig. 1b, Model 1) and another type with a strong (Fig. 1b, Model 2) directional tuning. Adjusting synaptic gain and ionic reversal potentials appropriately, both types of EMDs elicited identical responses of the model LPTC: When the pattern was moving in the PD of the cell, the cell depolarized; when the pattern was moving in the ND, the cell hyperpolarized (Fig. 1c, top left). During both PD and ND motion, $R_{\mathrm{IN}}$ dropped by about $15 \%$ of its initial value (Fig. $1 c$, bottom left). However, despite the identical signals, both types of EMDs elicited in the postsynaptic model LPTC under normal conditions, the models could be discriminated when inhibitory synapses were blocked (Fig. 1b, location of blockade indicated by shaded areas): (1) In the case of weak directional EMDs, the PD response increased, whereas the

Figure 1. $a$, Simulation of an LPTC receiving input from two arrays of EMDs tuned to opposite directions of motion and forming excitatory ( + ) and inhibitory $(-)$ inputs onto the dendrite of the cell, respectively. A VS-cell was three-dimensionally reconstructed from cobalt-stained material and was simulated as having only passive membrane properties. $b$, Two different types of EMDs were modeled: those with a weak (Figure legend continues) 
a
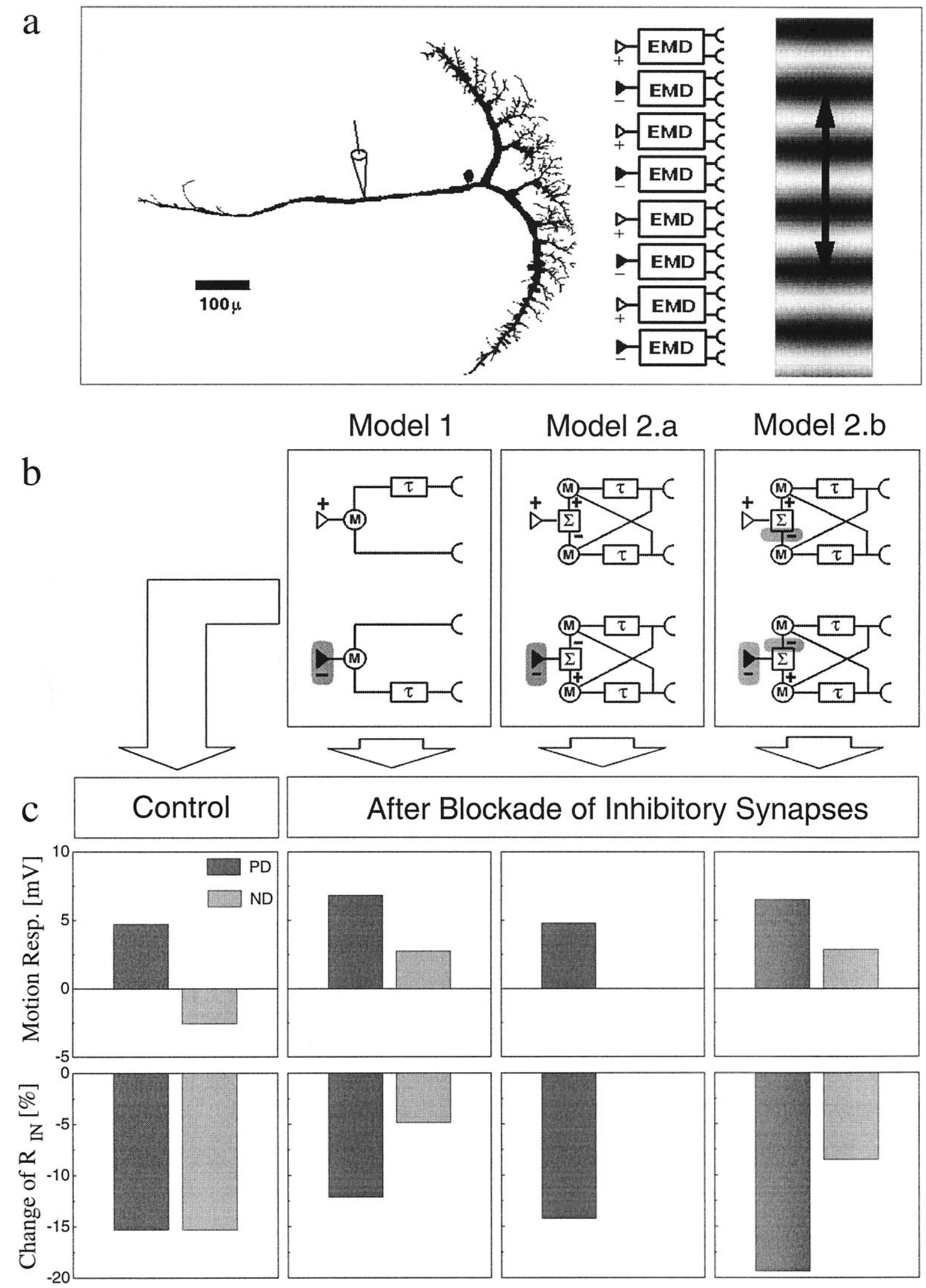

(Model 1) and those with a strong (Model 2.a and Model 2.b) direction selectivity. $c$, The responses (resp.) of the model LPTC were calculated for both types of EMDs under control conditions (left panel) and after inhibitory synapses were blocked (right panels, two different possible locations of blocking inhibition indicated by the shaded areas in the models in $b$ ). Under control conditions both models resulted in the same strong directionally selective motion response as well as in identical decreases of $R_{\mathrm{IN}}$ on the order of $15 \%$. When the inhibitory inputs were blocked, however, the different EMD models produced different fingerprints in the LPTC reactions, which allow discrimination between them experimentally. 
a
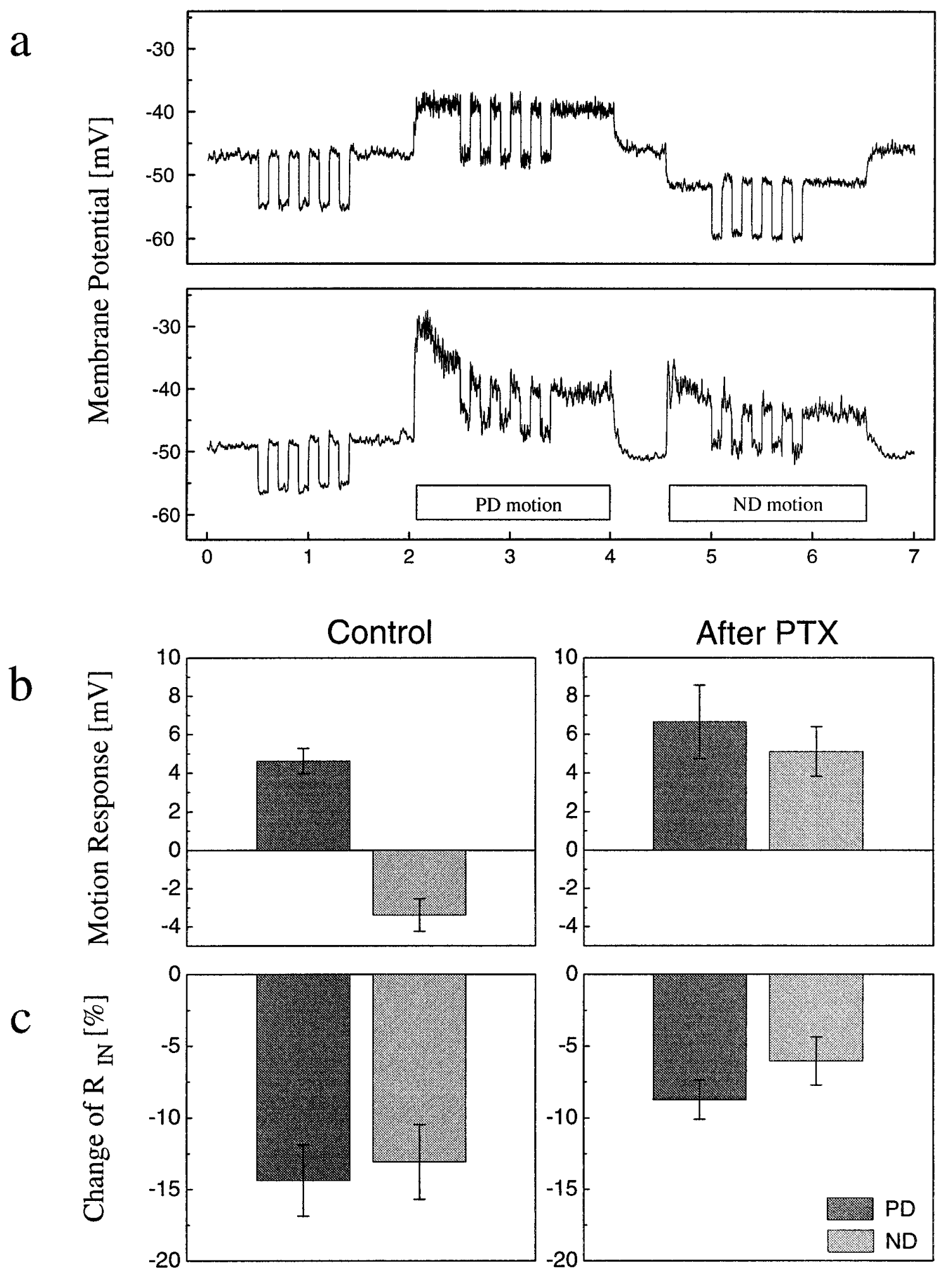

Figure 2. a, Intracellular recording from a VS-cell of the fly lobula plate before (top panel) and 10 min after (bottom panel) PTX has been applied to the hemolymph (mean of 10 sweeps). The bars underneath the lower response trace indicate the 2 sec time interval while a grating was moving in the $\mathrm{PD}$ and ND of the cell, respectively. During motion as well as while the pattern was at rest, $2 \mathrm{nA}$ pulses of hyperpolarizing current were injected into the cell to determine the input $R_{\mathrm{IN}} . b, c$, Motion response $(b)$ and motion-induced change of input resistance $\left(\left[R_{\mathrm{IN}}(\mathrm{motion})-R_{\mathrm{IN}}(\right.\right.$ rest $\left.\left.)\right] / R_{\mathrm{IN}}(\mathrm{rest}) \times 100\right)$ (c) before and after PTX had been applied. In normal fly saline, the cells depolarize in response to PD motion and hyperpolarize to ND motion. This graded shift of membrane potential is accompanied by a 13-14\% reduction of $R_{\mathrm{IN}}$ for both directions of motion. After PTX has been applied, the PD response increases, and the ND response inverses its sign. The motion-induced change of $R_{\mathrm{IN}}$ now only amounts to $<50 \%$ of its previous value for ND and $60 \%$ for PD motion. Data represent the mean \pm SEM of recordings from four VS-cells and three $\mathrm{CH}$-cells. 


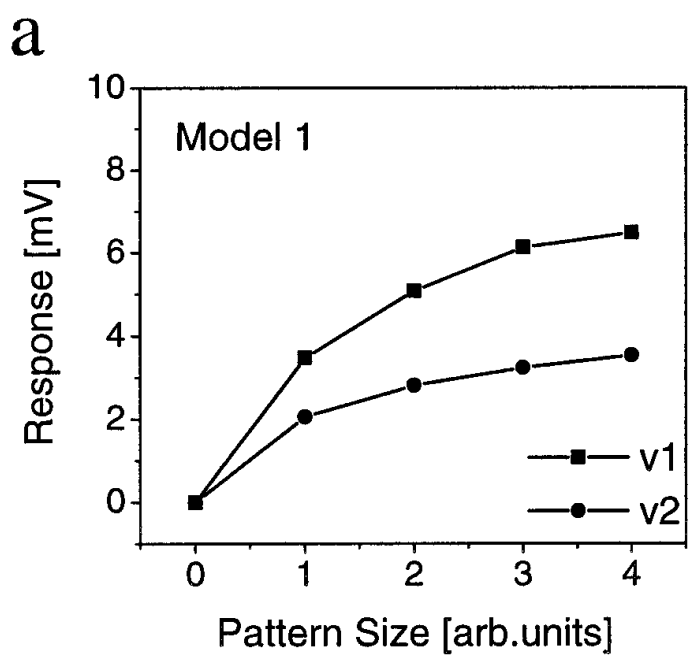

$\mathrm{C}$

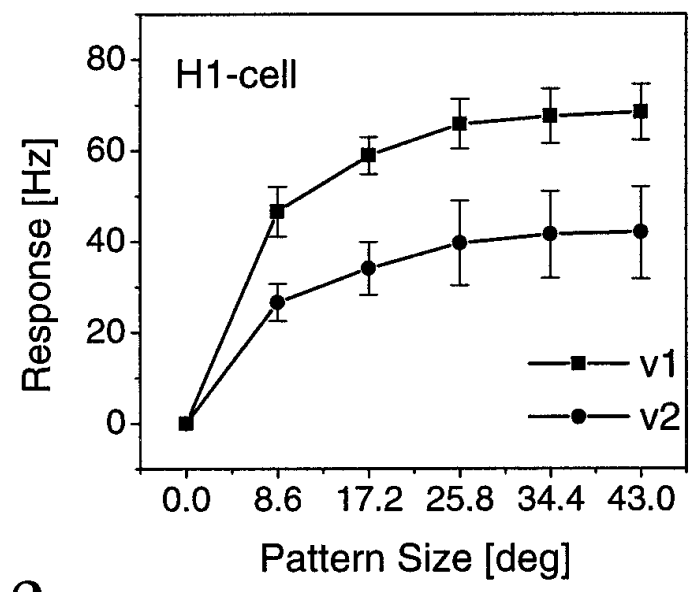

e

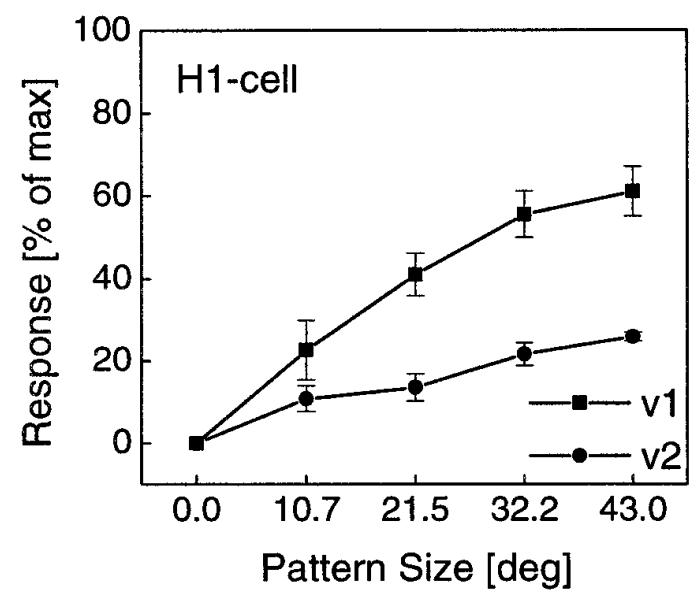

b

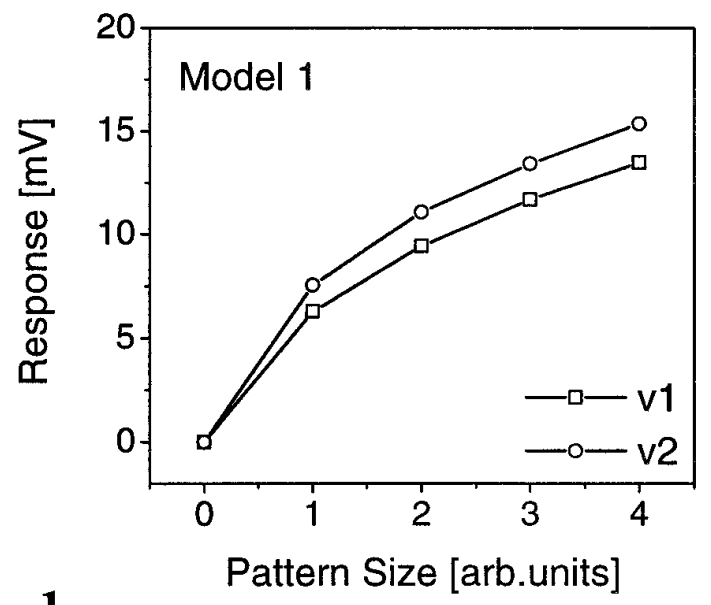

d
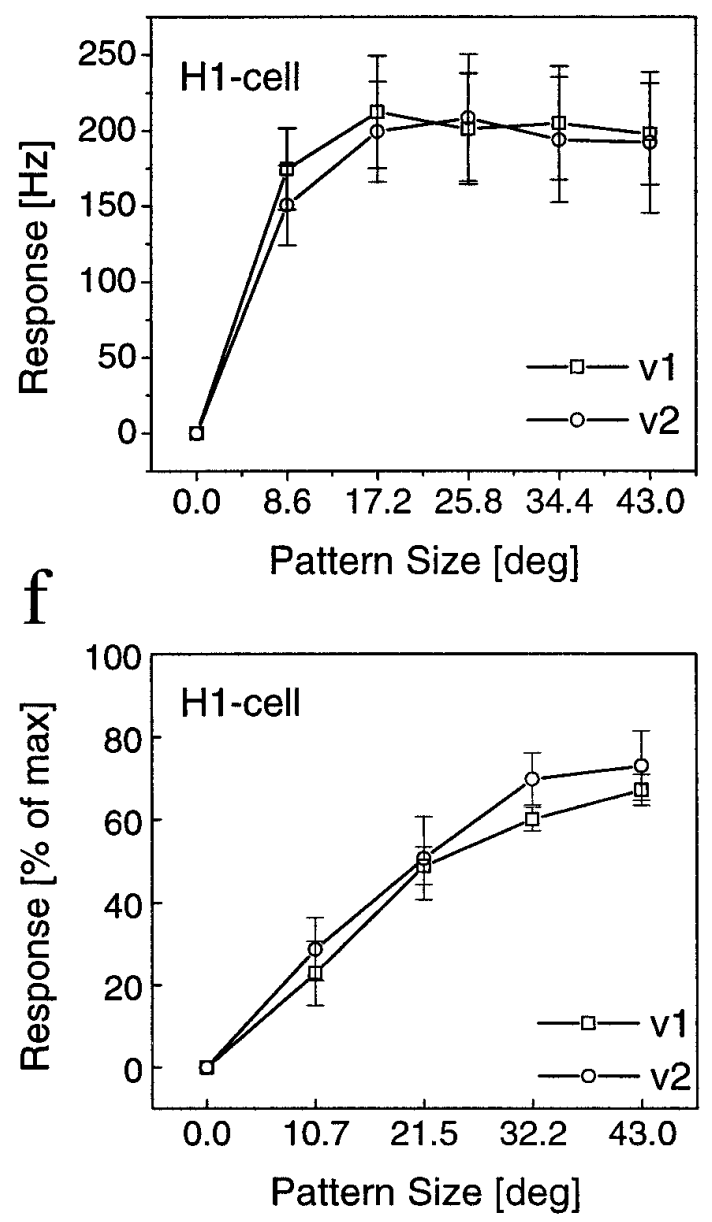

Figure 3. Gain control in model cell and real fly cell before and after blockade of inhibition. $a$, $b$, For the LPTC model, we used identical membrane parameters as in Figure 1. Inputs were driven by weak directional EMDs (Fig. 1b, Model 1). When stimulated by patterns of increasing size at two different velocities, the axonal membrane potential saturates at different levels (gain control). After blocking the inhibitory inputs, both velocities yield similar responses. $c-f$, Extracellular recordings of spiking LPTCs (H1-cells) before $(c, e)$ and after $(d, f)$ PTX application, using two different image velocities $(v 1=72 \% \mathrm{sec} ; v 2=360 \% \mathrm{sec}) . c$, In normal fly saline, the response increases with increasing stimulus size and saturates for each stimulus velocity at a different response level (gain control). $d$, After PTX application, the response increases significantly but now saturates at the same level for both velocities. Data represent the mean $\pm \mathrm{SEM}$ of recordings from four different animals. $e, f$, Same experiment as in $c, d$, but using low-contrast gratings this time. A high-contrast stimulus with full pattern size was additionally used to determine a maximum spike frequency of each cell. All responses are expressed as percentage of this value. After PTX application, the cells respond at about the same level to both velocities. However, the responses to v2 now is slightly stronger than to v1 (compare with simulation results in $a, b$ ). Data represent the mean \pm SEM of recordings from four different animals. 
ND response reversed its sign after blocking the inhibitory input synapses. This was accompanied by a reduced drop of $R_{\mathrm{IN}}$ during both PD and ND motion compared with the control situation (Fig. 1c, Model 1). (2) Strong directional EMDs lead to different LPTC responses after blocking inhibitory synapses. If only the input synapses to the LPTC were affected, both the response and the change of $R_{\mathrm{IN}}$ remained the same for PD motion as under control conditions. For ND motion, the response and the change of $R_{\text {IN }}$ became zero (Fig. 1c, Model 2.a). (iii) Additional blockade of inhibitory synapses within the EMDs resulted in the following scheme of LPTC response (Fig. 1c, Model 2.b): Similar to model 1 , the PD response increased, the ND response reversed its sign, and the change of $R_{\mathrm{IN}}$ during ND motion was reduced. However, in contrast to model 1, the increased PD response was accompanied by an increased change of $R_{\mathrm{IN}}$. Therefore, blocking inhibitory synapses in the system allows decision of whether the input signals to the fly LPTCs have a weak or strong directional tuning, even if the block might not be restricted to the input synapses of the LPTCs.

We tested these model predictions in intracellular recordings from fly LPTCs. In normal fly saline, PD motion led to a graded depolarization, whereas ND motion hyperpolarized the LPTCs. After PTX application, the PD response increased, whereas the ND response reversed its sign (Fig. $2 a, b$ ). Such a strong decrease of direction selectivity caused by the GABA receptor blocker has been reported previously for extracellular measurements of spiking LPTCs such as the H1-cell (Schmid and Bülthoff, 1988) as well as for other systems, e.g., rabbit retinal ganglion cells (Wyatt and Daw, 1976) or cells of the striate cortex (Sillito, 1977; Sato et al., 1995). The effects of PTX on the motion-induced change of $R_{\mathrm{IN}}$ as measured by injection of small hyperpolarizing current pulses were as follows. Before PTX application, PD and ND responses were accompanied by a significant drop of input resistance. After PTX application, this motion-induced change of input resistance was reduced to $<60 \%$ of its previous value for both directions of motion (Fig. $2 c$ ). Our finding of an increased PD response together with a decreased change of input resistance only agrees with model 1, i.e., the assumption of weak directional tuning of EMDS. This is in accordance with previous suggestions (Egelhaaf et al., 1990; Kondoh et al., 1995) and with recordings from T4-cells (Douglass and Strausfeld, 1996), a columnar cell type for which synaptic contacts onto LPTCs have been demonstrated and that might represent the output component of an EMD (Strausfeld and Lee, 1991). Thus, as is proposed for other motion-sensitive cells in vertebrates (Levick et al., 1969; Snowden et al., 1991), direction selectivity is significantly enhanced through the opponent action of local input signals on the dendrites in fly LPTCs.

Weak directional tuning of EMDs results in a joint activation of excitatory and inhibitory input by either preferred or null direction motion. This has the interesting functional consequence that the membrane potential of the postsynaptic cell is expected to saturate with increasing pattern size moving in the preferred direction of the cells at a level between the excitatory and inhibitory reversal potentials. Because for correlation-type local motion detectors the activation ratio of the excitatory and inhibitory input elements is a function of pattern velocity (Reichardt, 1987), the saturation level should vary with image velocity (Borst et al., 1995). This phenomenon, observed before in behavioral as well as electrophysiological investigations on flies (Hausen, 1982; Reichardt et al., 1983; Haag et al., 1992), is called "gain control." The reason for gain control can be seen by the following calcu- lation (Borst et al., 1995) approximating the membrane potential $(V)$ in an isopotential compartment $\left(E_{\mathrm{e}}\right.$ and $g_{\mathrm{e}}$ denoting excitatory reversal potential and conductance, respectively, subscript $\mathrm{i}$ for inhibitory, and $E_{\text {leak }}=0$ ):

$$
V=\left(E_{\mathrm{e}} g_{\mathrm{e}}+E_{\mathrm{i}} g_{\mathrm{i}}\right) /\left(g_{\mathrm{e}}+g_{\mathrm{i}}+g_{\text {leak }}\right)
$$

With increasing pattern size, $g_{\mathrm{e}}$ and $g_{\mathrm{i}}$ become large compared with $g_{\text {leak }}$, and the membrane potential tends toward a saturation level. Assuming $E_{\mathrm{e}}=-\mathrm{E}_{\mathrm{i}}$, this level can be expressed as:

$$
E_{\mathrm{e}} \times(1-c) /(1+c)
$$

with $c=g_{\mathrm{i}} / g_{\mathrm{e}}$ denoting the ratio of inhibitory and excitatory conductances being co-activated during PD motion. Obviously, without assuming additional mechanisms, gain control can only occur for weak directional EMDs. In correlation-type EMDs, the ratio $c$ is expected to depend on the image velocity $v$ in approximately the following way:

$$
c \cong \cos (R-\Phi(v / \lambda)) / \cos (R+\Phi(v / \lambda)),
$$

with $R$ denoting $2 \pi$ times the ratio of the sampling base of the EMD and the spatial pattern wavelength $\lambda$, and $\phi$ denoting the phase response of the temporal filter of the EMD.

We simulated the spatial integration properties of the model cell with weak directional EMDs at two different image velocities, with and without inhibitory synaptic input. With both excitatory and inhibitory input being intact, the model cell indeed showed gain control; with increasing pattern size the membrane potential saturated at different levels for both velocities, with the smaller velocity yielding larger responses (Fig. $3 a$ ). When the inhibitory input was blocked, both image velocities resulted in similar responses, and the response to the higher velocity was now slightly stronger than to the smaller one (Fig. $3 b$ ).

To verify these predictions experimentally we recorded the activity of a spiking LPTC, the H1 neuron, with an extracellular electrode and measured the responses to gratings moving horizontally along the preferred direction at two different speeds. In normal fly saline, the responses became larger with increasing pattern size and saturated at different levels for the two image velocities (Fig. 3c). After PTX application the responses were increased overall. Importantly, the neurons now responded with about the same strength to both velocities (Fig. $3 d$ ). Thus, in accordance with the simulation results, gain control was abolished after the inhibitory input to the LPTCs has been blocked. However, this effect could have also been attributable to a spike frequency saturation caused by the increased responsiveness of the cell after PTX application. We therefore repeated the experiments at low stimulus contrasts (Fig. $3 e, f$ ), including additional measurements of the maximum firing rate of the cell in response to high-contrast stimuli. Again, after PTX application, the cells responded with about the same spike frequency to both velocities. However, because of the low contrast stimulation, keeping the cell away from output saturation, more details of the response became visible: (1) in comparison with the control conditions, the response increase was more linear than before PTX application; and (2) the higher velocity yielding a smaller response before now resulted in a slightly stronger response amplitude than did the lower velocity. Both response characteristics were in close agreement with the simulation results (Fig. 3, compare $f$ with $b$ ). 


\section{DISCUSSION}

By measuring the motion-induced change of input resistance before and after the application of PTX, we have provided evidence that the input elements to the fly LPTCs exhibit only weak directional tuning. Consequently, the strong direction selectivity observed in the visual responses of fly LPTCs comes about by the opponent action of input elements. As a functional consequence the membrane potential of LPTCs saturates with increasing pattern size at different levels for different pattern velocities (gain control).

There are two critical questions arising in this context. The first question is to what extent the motion-induced change of input resistance can be attributed to synaptic conductance changes as was done here, or whether they reflect second-order effects such as voltage-gated conductances after synaptic activation. First of all, measurements of the specific passive membrane parameters of fly LPTCs (Borst and Haag, 1996) indicate a tight electrical coupling between the dendrite and axon of the cell, making synaptic conductance changes occurring in the dendrite clearly visible in the axon. Furthermore, we observed rather similar changes of input resistance during both preferred as well as null direction motion (Fig. 2c). If the drop of input resistance during visual motion was caused primarily by an opening of voltagegated conductances, a much stronger change of input resistance during preferred than during null direction motion would have been expected. However, visual motion leading to a depolarization has about the same effect on the input resistance as has visual motion that hyperpolarizes the cells. Therefore, the change of input resistance can safely be attributed to synaptic activity in a direct way.

Another important question pertains to the site of action of PTX. Because the drug was applied extracellularly to the lobula plate by either punctuating the neurolemma or direct pressure injection (see Materials and Methods), it could affect other GABAergic inhibitory synapses, too, besides the ones on the LPTC dendrites. When we added identical amounts of PTX to the hemolymph without punctuating the neurolemma, no effect on the visual response properties of the $\mathrm{H} 1$ neuron was observed, indicating a rather localized effect of the drug. As a further control, we also pressure injected PTX into the medulla instead of the lobula plate. Interestingly, besides some differences in the time course, these different procedures resulted in almost identical effects on the visual response properties of the H1-cell (data not shown). This can indicate either that, within the limits of our diagnostic tools, different sites of PTX action lead to similar results in the LPTC responses, or, alternatively, that PTX is rather free to diffuse within the optic lobes of the fly. Whatever the answer to these alternatives is, we know that (1) PTXsensitive GABA receptors do exist on LPTC dendrites and thus will be blocked by PTX, and (2) as can be seen by our simulation results (Fig. 1b, Model 2.b), additional action on presynaptic targets anywhere upstream of the LPTC dendrite results in an increased activity of the remaining excitatory input to the LPTC and consequently to an increased change of input resistance during PD motion. However, we observed a clear reduction of motion-induced change of input resistance after PTX application. This is only compatible with a rather specific effect of PTX restricted to the LPTC input synapses.

In this context, it is also of interest to consider the situation if the input elements had, in contrast to our conclusions, a full directional tuning. What would be the consequence? To explain both the large motion-induced changes of input resistance and the small amplitudes of LPTC visual responses, one had to postulate very small driving forces for the underlying ionic currents. As outlined in Materials and Methods, the value for the inhibitory current would be $13 \mathrm{mV}$ below, and the value for the excitatory current had to be only $24 \mathrm{mV}$ above the resting potential of the cell. Both values are highly unlikely, given the usual internal and external concentrations of the participating ions. Furthermore, fully directional input elements would lead to one fixed reversal potential in the postsynaptic cell, irrespective of pattern velocity. To explain gain control, one, therefore, had to postulate additional mechanisms for gain control in LPTCs.

In summary, as a functional consequence of having both the subtraction of opponent inputs as well as the spatial integration of these inputs implemented within one stage on the dendrites of the cells, their response can still signal changes in image velocity even under conditions in which the cells are spatially saturated. Apart from dynamic aspects of this signaling (Haag and Borst, 1996), such properties can be fully reproduced in passive compartmental model neurons by the change in balance of excitatory and inhibitory input. Because under free flight conditions the fly LPTCs are expected to be continuously stimulated by pattern motion fully covering their receptive fields, such a mechanism might be of ultimate importance for their proper functioning within the course control system of these animals.

\section{REFERENCES}

Borst A, Egelhaaf M (1989) Principles of visual motion detection. Trends Neurosci 12:297-306.

Borst A, Egelhaaf M (1990) Direction selectivity of fly motion-sensitive neurons is computed in a two-stage process. Proc Natl Acad Sci USA 87:9363-9367.

Borst A, Egelhaaf M (1992) In vivo imaging of calcium accumulation in fly interneurons as elicited by visual motion stimulation. Proc Natl Acad Sci USA 89:4139-4143.

Borst A, Haag J (1996) The intrinsic electrophysiological characteristics of fly lobula plate tangential cells: I. Passive membrane properties. J Comput Neurosci 3:313-336.

Borst A, Egelhaaf M, Haag J (1995) Mechanisms of dendritic integration underlying gain control in fly motion-sensitive interneurons. J Comput Neurosci 2:5-18.

Brotz TM, Borst A (1996) Cholinergic and GABAergic receptors on fly tangential cells and their role in visual motion detection. J Neurophysiol 76:1786-1799.

Brotz TM, Egelhaaf M, Borst A (1995) A preparation of the blowfly (Calliphora erythrocephala) brain for in vitro electrophysiological and pharmacological studies. J Neurosci Methods 57:37-46.

Douglass JK, Strausfeld NJ (1995) Visual motion detection circuits in flies: peripheral motion computation by identified small-field retinotopic neurons. J Neurosci 15:5596-5611.

Douglass JK, Strausfeld NJ (1996) Visual motion-detection circuits in flies: parallel direction- and non-direction-sensitive pathways between the medulla and lobula plate. J Neurosci 16:4551-4562.

Eeckman FH, Theunissen FE, Miller JP (1994) Nemosys: a system for realistic single neuron modeling. In: Neural network simulation environments (Skrzypek J, ed), pp 114-135. Boston: Kluwer.

Egelhaaf M, Borst A (1989) Transient and steady-state response properties of movement detectors. J Opt Soc Am A 6:116-127.

Egelhaaf M, Borst A, Pilz B (1990) The role of GABA in detecting visual motion. Brain Res 509:156-160.

Haag J, Borst A (1996) Amplification of high-frequency synaptic inputs by active dendritic membrane processes. Nature 379:639-641.

Haag J, Egelhaaf M, Borst A (1992) Dendritic integration of motion information in visual interneurons of the blowfly. Neurosci Lett 140:173-176.

Hausen K (1982) Motion sensitive interneurons in the optomotor system of the fly. II. The horizontal cells: receptive field organization and response characteristics. Biol Cybern 46:67-79. 
Hausen K (1984) The lobula-complex of the fly: structure, function and significance in visual behaviour. In: Photoreception and vision in invertebrates (Ali MA, ed), pp 523-559. New York: Plenum.

Kondoh Y, Hasegawa Y, Okuma J, Takahashi F (1995) Neural computation of motion in the fly visual system: quadratic nonlinearity of responses induced by picrotoxin in the HS and $\mathrm{CH}$ cells. J Neurophysiol $74: 2665-2684$.

Levick WR, Oyster CW, Takahashi E (1969) Rabbit lateral geniculate nucleus: sharpener of directional information. Science 165:712-714.

Reichardt W (1987) Evaluation of optical motion information by movement detectors. J Comp Physiol [A] 161:533-547.

Reichardt W, Poggio T, Hausen K (1983) Figure-ground discrimination by relative movement in the visual system of the fly. Part II: towards the neural circuitry. Biol Cybern 46:1-30.

Sato H, Katsuyama N, Tamura H, Hata Y, Tsumoto T (1995) Mecha- nisms underlying direction selectivity of neurons in the primary visual cortex of the macaque. J Neurophysiol 74:1382-1394.

Schmid A, Bülthoff H (1988) Using neuropharmacology to distinguish between excitatory and inhibitory movement detection mechanisms in the fly Calliphora erythrocephala. Biol Cybern 59:71-80.

Sillito AM (1977) Inhibitory processes underlying the directional specificity of simple, complex and hypercomplex cells in the cat's visual cortex. J Physiol (Lond) 271:699-720.

Snowden RJ, Treue S, Erickson RG, Andersen RA (1991) The responses of area MT and V1 neurons to transparent motion. J Neurosci 11:2768-2785.

Strausfeld NJ, Lee JK (1991) Neuronal basis for parallel visual processing in the fly. Vis Neurosci 7:13-33.

Wyatt HJ, Daw NW (1976) Specific effects of neurotransmitter antagonists on ganglion cells in rabbit retina. Science 191:204-205. 